\title{
Visualization of Large Amount of Spectra in Virtual Observatory Environment
}

\author{
Petr Šaloun ${ }^{1} \quad$ David Andrešič ${ }^{1} \quad$ Petr Škoda $^{2} \quad$ Ivan Zelinka ${ }^{1}$ \\ ${ }^{1}$ Department of Computer Science, Faculty of Electrical Engineering and Computer Science, VŠB-Technical University of Ostrava, \\ 17. listopadu 15, 70833 Ostrava-Poruba, Czech Republic \\ ${ }^{2}$ Astronomical Institute of the Academy of Sciences, Fricova 298, 25165 Ondrejov, Czech Republic
}

\begin{abstract}
This paper presents overview of new features so far prepared for new version of spectral analysis tool SPLAT-VO that allows to retrieve a large amount of spectra (and other data) based on its characteristics by detailed querying a virtual observatory's resources. The overview is focused on enhancements of user experience, work with simple application messaging protocol (SAMP) and other interoperability that improves work with global list of spectra, plot window and analysis menu.
\end{abstract}

Keywords: SPLAT-VO, virtual observatory, spectra, simple application message protocol (SAMP), flexible image transport system (FITS).

\section{Introduction}

The large amount of spectra requires capable tools for selecting by varied criteria, processing and visualization. Large amounts of data exist, and today's tools that the astronomers are using (like SPLAT-VO) need to be adjust accordingly.

Virtual observatory (VO) is a collection of interoperating data archives and software tools which utilize the internet to form a scientific research environment in which astronomical research programs can be conducted. VO offers huge data-sets with thousands of fuzzy interconnected attributes for researcher, but it is necessary to select the proper one and show them.

SPLAT is a shortcut for spectral analysis tool ${ }^{[1]}$, and it is a tool for displaying, modifying and analysing astronomical spectra, for example. The examples can be seen in Figs. 13 , respectively. SPLAT was developed in 2003 as a part of Starlink (and its STARJAVA package) project ${ }^{[1]}$. The Starlink was closed down in 2005 and took over by joint astronomy centre ${ }^{1}$ that relicensed some of its parts under GNU/GPL licence in $2006^{[2] 2}$.

During its development, the SPLAT was extended to include facilities that allowed an interoperability with the

\footnotetext{
Regular paper

Special Issue on Recent Advances on Complex Systems Control, Modelling and Prediction II

Manuscript received August 19, 2013; accepted October 11, 2013

This paper was supported by Agency of the Czech Republic-GACR (No. P103/13/08195S), the Development of Human Resources in Research and Development of Latest Soft Computing Methods and Their Application in Practical Project, Operational Programme Education for Competitiveness (No. CZ.1.07/2.3.00/20.0072), ESF, State Budget of the Czech Republic, SGS (No. SP2013/114), VŠBTechnical University of Ostrava, Czech Republic.

${ }^{1}$ www.jach.hawaii.edu

${ }^{2}$ GNU General Public License-www.gnu.org/licenses/gpl3.0.txt
}

virtual observatory ${ }^{[1] 3}$. The $\mathrm{VO}$ is a collection of astronomical archives and software tools that utilizes the internet to allow international collaboration and scientific research $^{[3]}$. SPLAT itself uses the simple spectra access protocol (SSAP) ${ }^{4}$ for obtaining the spectra from SSAP servers and simple application messaging protocol (SAMP) ${ }^{5}$ to interoperate with other SAMP-compatible tools like tool for operations on catalogues and tables (TOPCAT) ${ }^{6}$.

This paper completes our work, which is about to be accepted by developers and research community of SPLAT, our previous paper ${ }^{[4]}$ described our first results in this area.

Since 2012, SPLAT-VO has been developed by the German Astrophysical Virtual Observatory (GAVO) ${ }^{7}$ in cooperation with the Astronomical Institute of the Academy of Sciences of the Czech Republic ${ }^{[1] 8}$. Its development is currently focused on implementing new VO standards. The need to extend the scientific features that do not immediately relate to the $\mathrm{VO}$ and to fix some of the SPLAT's bugs or deficiencies resulted in a bachelor thesis at VŠB-TUO ${ }^{9}$ supervised by Petr Škoda of Astronomical Institute of the Academy of Sciences of the Czech Republic. This thesis scoped some changes to SPLAT-VO as described below.

In the near future, after dealing with coordination and unification of SPLAT's development, we intend to continue to add new features and implement new VO standards so that the SPLAT can be a feature-rich, user-friendly and even more portable scientific tool and reference implementation of new VO standards.

\footnotetext{
${ }^{3}$ Standardized, coordinated and developed by international virtual observatory alliance (IVOA) - www.ivoa.net

${ }^{4}$ www.ivoa.net/Documents/SSA/

${ }^{5}$ www.ivoa.net/Documents/latest/SAMP.html

${ }^{6}$ www.star.bristol.ac.uk/mbt/topcat/

${ }^{7}$ www.g-vo.org

${ }^{8}$ www.asu.cas.cz

${ }^{9} \mathrm{VŠB}$ - Technical University of Ostrava, www.vsb.cz
} 


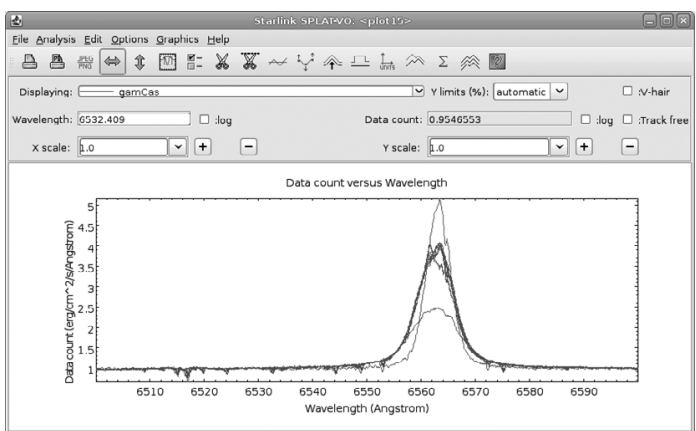

Fig. 1 SPLAT-VO at work

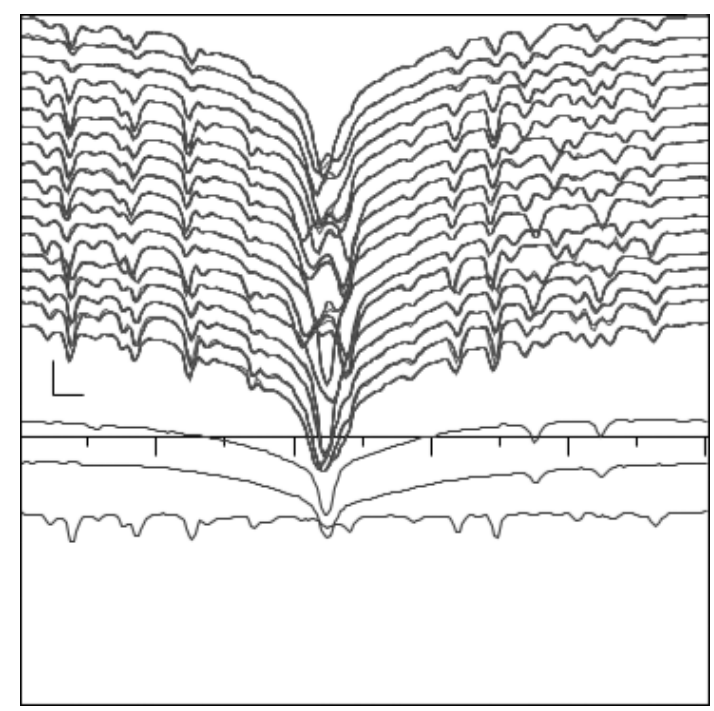

Fig. 2 Double star Her 96 and its $H_{\alpha}$ spectral line

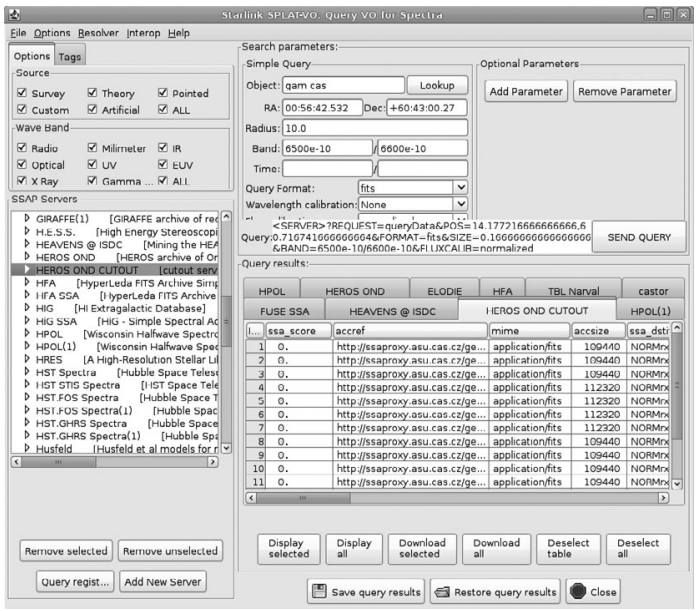

Fig. 3 SSAP window in SPLAT-VO for querying virtual observatory

\section{New features}

Besides the strictly virtual observatory related changes, which will allow the user even more detailed searching for spectra, the upcoming version of SPLAT-VO focuses on the user experience, interoperability with other tools and work with the spectra itself. On the next few pages, we describe these new features. We believe that all together will make your work with SPLAT more enjoyable.

\section{$2.1 \quad$ Plot window}

The purpose of plot window is quite simple: Plot every spectrum selected in the global list of spectra and allow the user to perform analysis on it. Every plot window can contain one or more spectra and when working with SPLAT, the user can open many plot windows with many spectra plotted in it.

\subsubsection{Added visual spectrum selection capability}

So far, when the user worked with multiple spectra in one common plot window, he has had a limited possibility to select just one spectrum for further actions. For example, selecting noisy spectrum and its deletion from plot window could be done only by trial-and-error procedure, and it is quite problematic and time-wasting.

In the upcoming version, the user can click by mouse inside the plot window and SPLAT will automatically select the closest spectrum. The spectrum is selected in the local spectra drop-down menu above the plot itself and the global list of spectra in the main window. From there, it can be easily deleted or modified for plotting and analysis.

The actual selection is done by getting the $[x, y]$ coordinates of mouse click, which are then transformed to coordinates with the plot's $[0,0]$ as beginning. Then, the algorithm searches for all spectra with $x$ coordinates containing the $x$ coordinate of the mouse click. For this, the native (platform dependent) AST library ${ }^{10}$ from the original Starlink project is used. Each spectrum is represented by series of $[x, y]$ coordinates, each coordinate represents one value. The AST library transforms this discrete list of values to a continuous line that can be seen in the plot window. This set of transformed coordinates is used by the selection algorithm. So when any spectrum is detected on the $x$ coordinate, the algorithm will again use the modified binary search algorithm ${ }^{11}$ to select the closest spectrum on the $y$ axis. This is quite similar to the procedure on $x$ axis, the coordinates (transformed via AST) are used to locate the closest match of each $x$-colliding spectrum's $[x, y]$ coordinates to the mouse click coordinates.

The selection of the spectrum is quite reliable. To ensure this, the selected spectrum is immediately highlighted by few-seconds blinking in inverted color (It will be described

\footnotetext{
${ }^{10}$ A library for handling world coordinate systems in astronomy, http://starlink.jach.hawaii.edu/starlink/AST

${ }^{11}$ The usual binary search algorithm searches for the exact match of searched item in the sorted list by getting closer to it through dividing the current interval (where the first interval is $0, \cdots, n$ ) into two. Then, by comparing the searched value to the subinterval's borders, it divides the first or second subinterval in the next iteration. The modified algorithm does not look for the exact match, but gets as close as possible to the searched item. Then, it compares the first item on the left side with first item on the right side. Finally, it selects the closest to the searched one.
} 
in Section 2.2.3).

The usage of modified binary search algorithm is crucial when considering the user's computer performance, so it will not cause any slow-down of SPLAT's interface.

\subsection{Global list of spectra}

The global list of spectra is a part of the SPLAT's main window. It is a container (JList) for every spectrum opened no matter from which source. Since it is a source of each spectrum in $\mathrm{SPLAT}^{12}$, every major component of SPLAT is tied to it.

\subsubsection{Saving in flexible image transport system (FITS)}

Previous versions of SPLAT were able to save its global list of spectra only in their native binary format (More technically, it is just a serialized object representing the JList with individual spectra's instances). But sometimes, there may be a need to save that list in a more universal, standardized format. For example, SPLAT allows to perform many operations on spectra, which results in a new spectrum object that is added to a global list of spectra (e.g., cutting a part of spectrum). Saving the list of such spectra to a standardized format would then allows its opening in another tool and continuing to work with it in a way the SPLAT was not designed to.

Therefore, the upcoming version of SPLAT-VO will allow the user to save the global list of spectra to a universal FITS format. The FITS format is multipurpose format for storing the scientific data. It is primarily designed and used for astronomy ${ }^{[5]}$. The FITS allows storing multiple data of multiple type in it. For example, it can contain the image of a star and its spectrum (or spectra) with corresponding metadata. This is done by a concept of extensions. For a programmer, the extension can be described as an instance of a class, where class itself is a type of extension. We recognize these basic types of extensions as follows ${ }^{[6]}$ :

1) IMAGE: Provides a means of storing a multidimensional array similar to that of the FITS primary header and data unit.

2) TABLE: ASCII table extension type which contains rows and columns of data entries expressed as ASCII characters.

3) BINTABLE: Provides a more flexible and efficient means of storing data structures than those which are provided by the TABLE extension type.

Every FITS file consists of one primary header data unit (HDU) and of an unlimited number of extension HDUs. Every HDU contains (besides its actual data) the ASCII header with corresponding metadata as key/value pairs, where the most important keys are standardized and understood by FITS readers (e.g., extension key identifying the type of the current extension).

\footnotetext{
${ }^{12}$ It actually works as a proxy design pattern: No matter what the source or format of the spectra is, each one can be used transparently and in the same way because it is transformed to an internal SpecData object.
}

In the upcoming version of SPLAT, every spectrum will be represented by its own IMAGE extension. So when the global list contains (let's say) five spectra, it will result in an FITS output with five IMAGE extensions (one for each spectrum). The only thing that the user will have to do for this is to select the newly added FITS/IMAGE format in a dialog window for saving the global list of spectra, instead of the previous proprietary format.

The only disadvantage compared to the proprietary format is that it would not keep the settings like color or strength of the line, etc. This is primary because of the fact that these metadata would have to be added to its headers and FITS is designed to be a universal format. So adding these SPLAT-specific metadata seems to be a little bit questionable at this moment (There is a way, if this feature would be required in practice).

We are also planning to add the support for another types of FITS extensions (e.g., BINTABLE), as well as other formats like VO-Table.

SPLAT will also be capable of reading the global list in FITS format. This may be caused by a feature more closely described in Section 2.2.2. Unlike saving the global list, for the opening of it, the input FITS is not limited only to an FITS/IMAGE extension. But it can generally be any valid FITS file.

\subsubsection{Improved support for multi-HDU FITS files}

In the previous versions, SPLAT was (simply put) reading only the first HDU found in the FITS file. But FITS can contain many HDUs in a form of an extension as described in Section 2.2.1. These extensions were basically inaccessible in SPLAT.

In the upcoming version, SPLAT will be able to load all HDUs located in the input FITS file. Each HDU will produce a spectrum item in the global list of spectra referencing the corresponding FITS header. This means that features like showing the FITS headers or ordering by its metadata (contained in FITS headers) will automatically work with this new feature. Therefore, SPLAT will become a truly general-purpose, feature-rich FITS viewer and editor. More importantly, the ability to access all FITS headers (or more precisely its metadata) also improves SPLATs spectral analysis capabilities.

Because of gentle refactoring of SPLAT's architecture, one source (e.g., file) provides one spectrum. Newly, SPLAT expects the multiplicity 1:M. So when there will be a need in the future to add a similar functionality (to load more spectra from one source file), SPLAT will be ready for it.

\subsubsection{Highlighting the spectrum after selection}

When user clicked on a spectrum in global spectra list and this spectrum was plotted to a window(s) with multiple spectra, he didn't know which one it is.

In the upcoming version, when user clicks on a spectrum in global spectra list, the spectrum gets immediately highlighted in all plot windows that it is plotted in. The highlighting has a form of a few-seconds blinking in an inverted color and is performed sequentially (one plot window by 
one).

The algorithm used to do this is the same as in the case of visual spectra selection as briefly described in Section 2.2.1. It is designed to not cause any slow-down of SPLAT's interface. This is done by listening the mouse-click event and performing all the iterations of inverting the spectrum's plot color, repainting the components and sleeping in special threads. Therefore, SPLAT's user interface does not get frozen during the sleep phase and is not touched otherwise. The highlighting threads are also synchronized, so one starts highlighting after the previous is finished and the user gets a perfect sequential overview of the spectrum's plots.

As for the inversion, the spectrum's color is treated as java.awt.color ${ }^{13}$ in its integer expression. Basic colors red $(R)$, green $(G)$ and blue $(B)$ are encoded in this way:

1) Red: bits 16-23.

2) Green: bits $8-15$.

3) Blue: bits $0-7$.

For inverting the color, we need to decode each basic color (this can be done by class's getters), invert it by subtracting this decoded value from 255 (range for each color), and put it back to its decade through multiplying it by the corresponding offset ( 1 for blue, 256 for green and 65536 for red). The final inverted color is then a sum of these inverted components.

\subsection{Analysis menu}

The plot window's analysis menu is a doorway to SPLAT's arsenal of analytic features like cutting regions from spectra, fitting, interpolation, filtering and more. Many of these functions are still based on native, platform dependent libraries of the original Starlink project.

\subsubsection{Cut window: Saving the ranges}

In the "Cut regions from spectrum" window, user could simply read ranges of regions from a local file (feature available under "File" menu). But what if the user performed a visual selection of ranges from a currently plotted spectrum and wanted to save it to a local file?

Since the upcoming version, SPLAT can save the currently defined ranges to a local file (feature is available under "File" menu) in the same format as the reading feature expects, that is one range (with a white space between its beginning and ending value) per line:

\# Generated by SPLAT-VO

\# Range 1

8601.1778621 .531

\# Range 28729.0268748 .744

Adding this feature was the simple, since SPLAT already possessed this feature. But it was just used to a different purpose. Thanks to this, it consisted just from adding the

\footnotetext{
${ }^{13}$ docs.oracle.com/javase/1.4.2/docs/api/java/awt/Color.html
}

new item to the menu and binding the existing function to it.

\subsubsection{Cut window: Performing on multiple spec- tra}

Until now, when user defined all the wanted regions and performed the cut action, SPLAT cuts the regions only from the currently active spectrum.

In the upcoming version, this window will possess a table of all currently plotted spectra in the corresponding plot window (see Fig. 4). User then can select several of them (or use buttons for selecting and deselecting all the spectra) and perform cuts (or any other action in this window) on all the selected spectra.

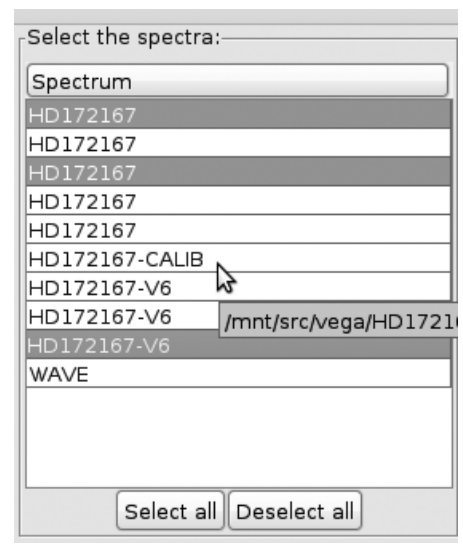

Fig. 4 New universal table for spectra selection

Adding this feature was quite straightforward. It consisted from creating a new Java class extending the standard Java's JTable, creating its appropriate cell-render class and passing a JList with all currently plotted spectra to it. The JList's items then get rendered to this table. One of the new methods returns a list of all the selected spectra, which is only a step from modifying the action functions (e.g., cut) to iterate over it.

This new spectra selection table is written very generally, so adding a similar feature any time in the future will be quite easy.

\subsection{SAMP-compatible tools interoperabil- ity}

Simple application messaging protocol (SAMP) is universal, XML-based, event-driven messaging protocol for exchanging control and data information and thus allows the (not just astronomical) software to interoperate ${ }^{[7]}$.

Usage of SAMP allows building an event-driven publish/subscribe messaging system with both synchronous and asynchronous communication. The whole communication is centralized. All elements communicate with an SAMP hub that takes care of routing the messages ${ }^{[7]}$. In the typical scenario, an application interested in using the SAMP looks for a SAMP hub using the appropriate discovery mechanism (the hub itself - using the same mechanism - guards that there is only one SAMP hub running), 
connects to it and sends to it metadata that contains its name, type of messages that it is interested in and asks the hub for information about other clients. Then, when some hub client (publisher) has a message to publish, it will notify the hub that will decide (based on type of the message) which registered clients (subscribers) will be notified as well. When a subscriber is notified, it can ask the hub for the message. The hub will then require the message from the publisher and sends it to a subscriber.

For having a better idea about SAMP's purpose and capabilities, imagine an (e-)conference (or any other form of real-time cooperation) where one user performs an action using his client application (e.g., the user clicking on a spectrum in TOPCAT). Other users with their completely different client applications can see the same information and result but in a form native for their client applications (e.g., spectrum sent by TOPCAT plotted in SPLAT) in the same time.

\subsubsection{All SAMP spectra to the same plot window}

In the previous versions of SPLAT, all spectra received via the SAMP protocol got opened in their own plot windows. Very often, it would be useful or even necessary for analysis to have them plotted in the same window. But for this, the user had to do it manually.

As stays in the title, the upcoming version will be able to do this for the user automatically. We added new checkbox under the "Interoperability" menu (see Fig. 5) that can be used to switch between the current state (each spectrum to its own plot window) and plotting all the spectra received from SAMP to their common plot window.

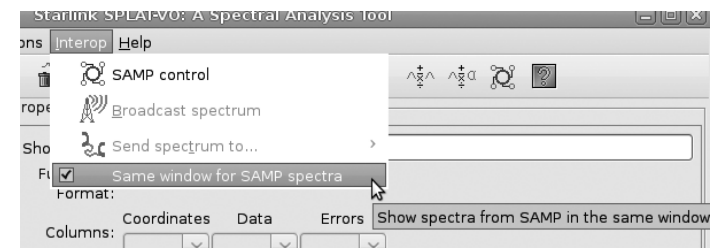

Fig. 5 Enabling the new feature plotting all SAMP spectra to the same window

Technically, it is done by adding the new "source type" attribute into the spectrum's I/O process and mapping every plot window to its initial spectrum's source type (In the case of SAMP, this reference is removed from every possibly opened SAMP-containing plot window when user disables this feature, so it allows to plot one bulk of spectra received from SAMP to one window and another bulk to another window). Then, when the new spectrum is received (but this may vary in the future) and the feature is switched on, SPLAT will first look for an existing plot window that is mapped to SAMP source type. And if it is found, it will plot the spectrum into it (and properly resizes it as usual).

As mentioned, this feature was added using the new attribute for spectrum's I/O process defining the spectrum's source. So far, this attribute is bound just to SAMP protocol, but it allows simple modifications of I/O behaviour based on the spectrum's source in the future by simply writing the extra logic for non-default source type.

\section{Bug fixes}

Since the SPLAT is based on Starlink's libraries, which has been used for many years, discovery of new bugs is limited to less critical parts of SPLAT. Yet, for some users, they make the SPLAT unusable. We are now in the process of removing those we know about, but we welcome new bug reports.

\subsection{SSAP query window}

In some cases, when user tried to open the SSAP query window to search for spectra via SSAP protocol, the only thing that he saw was a window with a list of queried SSAP servers. He could not enter any value or parameter.

This was caused by a NullPointerException in SSAP query window initialization. After hotfixing it and consulting with Margarida Castro Neves of Heidelberg University, the problem was located in a PropertyChangeSupport property initialization. Since the JPanel already has it, it was fixed by removing it and refactoring the rest of the code to use the JPanel's one.

\section{Features for developers and experi- mentators}

It will still take some time before the new version's release, but if anyone would like to try it now or participate on testing, here is the essential build "HOWTO" and short description of its innovations.

\subsection{Java 1.7 compatibility}

By this is meant SPLAT's compilation and building compatibility (Runtime compatibility is generally guaranteed by Java itself). Until now, Java 1.6 has been used to build SPLAT. Building with Java 1.7 caused build failures for several reasons described below.

Still, building with Java 1.7 keeps SPLAT compatible with Java 1.6 runtime environment.

\subsubsection{JSAMP's JAR sign algorithm}

When built with Java 1.7, SPLAT was throwing java.lang. SecurityException: invalid SHA1 signature file digest - exception during its start. The reason is the changed sign algorithm for JARs in Java 1.7. This problem can be fixed by adding the digestalg="SHA1" parameter to jarsigner, which will force using the previous sign algorithm.

Since STARJAVA/SPLAT is being built by the ant utility, the digestalg parameter should come to the JSAMP's build.xml file. It also requires modification

\footnotetext{
${ }^{14}$ As said before, our current primary goal is to unify the development since the SPLAT's source codes are located in several repositories. Version described in this article can be found at Github: http://github.com/and146/SPLAT-ARI/
} 
of STARJAVA/SPLAT's customized ant (added some STARJAVA-special ant targets) in the sense of adding the support for these new parameters to its source, since they are available from higher versions of ant that STARJAVA contains.

\subsubsection{Java 1.6 backward compatibility}

By default, SPLAT's binaries are runnable under a version of Java that has been used to compile it. Keeping the backward compatibility required adding the target parameter for javac in build.xml file of SPLAT and all of its required tools.

\subsubsection{Other modifications}

This section closely relates to Section 4.2 and usage of a build script. By default, this script builds the entire STARJAVA package so when Javac 1.7 is used, it throws errors in other tools as well (Some of them are not required to successfully build the SPLAT). This is a short list of modifications required to successfully use the Javac 1.7, yet none of them is so far a part of its original distribution.

As first, Frog was using a Sun's proprietary (not Javastandard) library for exporting the image (JPEGCodec and JPEGImageEncoder), which was removed in Java 1.7. This needed to be rewritten to use its standard equivalent (ImageI0).

Then, TOPCAT is using in SyntheticColumnQueryWindow class getters and setters for a parameter called type. Unfortunately, this class extends the Java's java.awt.Windows since Java 1.7 contains an internal enum called Type. This was causing the incompatible return data type of uk. ac.starlink. topcat. SyntheticColumnQueryWindow.getType() exception during building with javac 1.7 .

And finally, on some systems the build could fail on invalid encoding of TAMFITS's source (nom.tam.FITS is Java library for working with FITS files). This was fixed by explicit adding the encoding="iso-8859-1" parameter to its build.xml file.

\subsection{Building using the buildscript}

Basically, until now the only way to build SPLAT was (at least in some way) following the instructions in the original STARJAVA's README file. To automatize this (and to add some more "shortcuts"), the build script was created.

The build script (_builder.sh) can be used on every Linux system for automation of STARJAVA's build process. It performs some system checks and allows quite userfriendly way to build only a subpart of STARJAVA. The user can also specify a version of Java used for building the result package. For full list of its capabilities, run it with the --help parameter.

\subsubsection{Source}

As said before, our current primary goal is to unify the development since the SPLAT's source codes are located in several repositories. Version described in this article can be found at Github: https://github.com/and146/SPLAT$\mathrm{ARI} /$.

\subsubsection{Prerequisites}

Checks for most of them are included in the build script, so only a short list follows:

1) Java advanced imaging (JAI).

(http://download.java.net/media/jai/builds/release/1_

1_3/INSTALL.html)

2) Java development kit $(\mathrm{JDK}) \geqslant 1.6$.

(http://www.oracle.com/technetwork/java/javase/down loads/index.html)

4.2.3 Build

The actual build is quite straightforward (demonstrated on GNU/Linux):

\# first, download the source

(starjava_parent) \$ git clone । git://github.com/and146/SPLAT-ARI/

\# enter the newly created directory and run the

\# build script

(starjava_parent) \$ cd SPLAT-ARI

(SPLAT-ARI) \$./_builder.sh

\# the binaries should be located in

\# (starjava_parent)/bin:

(SPLAT-ARI) \$ ../bin/bin/splat/splat

\subsubsection{HOWTO: Creating an installation package}

For enthusiasts playing with SPLAT's source code, it could be nice to have an individual installer's JAR for each build they create. This section describes how to create an installation package of SPLAT-VO. It is based on Peter Draper's HOWTO as well as Margarida Castro Neves's modifications of it.

Logo, some scripts and some other useful files are contained in a file called extra_files.tar. This is a file created by Peter W. Draper and is generally not public. Yet, these files should not be necessary unless you want to create a uniform, 1:1 installer package.

This HOWTO describes creating a package on a GNU/Linux OS.

1) Prerequisites

Starjava's source codes. Since some utilities from the original Starjava distribution are required, the first step is to get the source code of the original Starjava containing the SPLAT-VO. We can get it and build it using the build script as described previously in Section 4.2 (if we intend to create a package with modified version) or we can download and build the currently official source codes from http://starlink.jach.hawaii.edu/starlink/ and proceed as described in its README file.

It should be noted that the development team will change this very soon (probably right after finishing this thesis) because of merging and unification of the source code and its relocation to a Git repository. The only difference then will be a different URL with the source codes and the way to build it.

We will need the C-shell interpreter $\left(\operatorname{csh}\right.$ or $\left.\operatorname{tcsh}{ }^{15}\right)$ in-

\footnotetext{
${ }^{15}$ http://www.tcsh.org/
} 
stalled.

2) IzPack

For actual creating the package, the $\mathrm{IzPack}^{16}$ is used. IzPack is a famous tool for creating a platform-independent installer packages on Java platform.

a) Run IzPack's installer

$\$$ java -jar IzPack-install-4.3.5.jar.

b) After the installation, we will need to add the IzPack's bin directory to the \$PATH, or make the necessary symbolic links:

\# In -s /opt/IzPack/bin/compile/usr/bin/compile.

3) extra_files.tar. Extract the extra_files.tar scripts to the parent of (starjava) directory.

4) Prepare the environment.

a) Prepare the source codes in (starjava) directory (clean, checkout repository, etc.).

b) Run this script from extra files tarball

extra_files.tar

(starjava_parent) \$ source setup.

This script prepares the environment for building SPLAT. All the paths in the script should be adapted to your system.

c) As an alternative, the next command should be enough at least for already built binaries (see 1)):

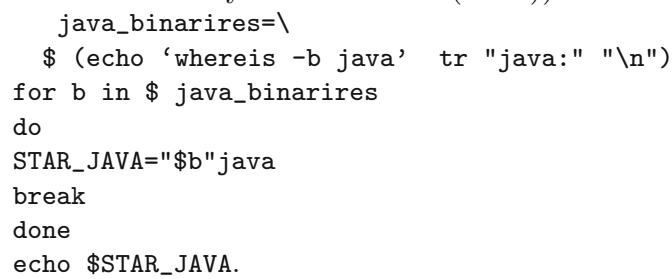

5) Installing only the SPLAT relevant stuff

a) If we did not build the $\mathrm{Ant}^{17}$ and the rest of the sources, now it is time to do so.

b) Run this: (starjava) \$./scripts/targetdeps \ splat install.

c) Test it:

(starjava) \$ cd $\cdots$

(starjava_parent) \$./bin/splat/splat.

6) Update documentation

a) Generate the sun243.ps file.

b) Update the splat/src/docs/splat.news.

c) Update help files if necessary.

7) Remove all the unnecessary files

(starjava_parent) \$./removed_files.lis.

8) Finalization before creating the installer

a) Update release version in install.xml, which should be copied to the release directory.

b) Make sure all the scripts have execute permission.

9) Build installer JAR file by running this command:

(starjava_parent) \$./doit.csh.

Or as an alternative, this is a manual way (in the same directory as above):

\$ compile install.xml -b . -o . / splat-vo.jar ।

$-\mathrm{k}$ standard.

\footnotetext{
${ }^{16}$ http://izpack.org/

${ }^{17}$ http://ant.apache.org/
}

For this, IzPack has to be installed, and it is bin directory needs to be in the system's \$PATH variable (to execute the compile command).

1) Unix_shortcutSpec.xml;

2) ShortcutSpec.xml;

3) Starlink.gif (should also be copied to the release directory).

They are all part of extra_files.tar.

And that is it! You have successfully created an installer package of SPLAT-VO. Testing the release and finalization should come next, as well as some other publishing procedures if you are a part of a developer team.

\section{Conclusions}

Being more focused on user, experience should allow current users to work with SPLAT more effectively (especially with a large amount of spectra). For more reserved astronomers that so far had a reason to avoid SPLAT, we hope we can provide a reason or reasons to give it a try.

In the near future, we are planning to work on (beside strictly VO-related parts, that are currently being developed at Heidelberg University) user-defined line lists, working with spectrum's header information in plot window or supporting more file formats. And at least, some of these changes should be a part of the next release as well.

\section{Acknowledgement}

We appreciate the help of Margarida Castro Neves, Markus Demleitner, Peter W. Draper and Mark Taylor. Consultation and other hints about SPLAT they kindly provided were essential for this work.

\section{References}

[1] P. W. Draper. Starlink SPLAT-VO, [Online], Available: http://star-www.dur.ac.uk/ pdraper/splat/splat-vo/, 2013

[2] Joint Astronomy Centre. Starlink, [Online], Available: http://starlink.jach.hawaii.edu/starlink, 2013.

[3] International Virtual Observatory Alliance. International Virtual Observatory Alliance, [Online], Available: http: //ivoa.net/, 2013.

[4] P. Šaloun, D. Andrešič, P. Škoda, I. Zelinka. Upcoming features of SPLAT-VO in astroinformatics, In Nostradamus 2013: Prediction, Modeling and Analysis of Complex Systems, Advances in Intelligent Systems and Computing, Springer, vol. 210, pp. 475-486, 2013

[5] M. Donahue, T. Kimball. Fits file format. HST Data Handbook, [Online], Available: http://www. stsci.edu/documents/dhb/web/c02_datafiles.fm2.html, 2013.

[6] W. D. Pence. FITS Extension Names, [Online], Available: http://fits.gsfc.nasa.gov/xtension.html, 2013. 
[7] M. Taylor, T. Boch, M. Fitzpatrick, A. Allan, J. Fay, L. Paioro, J. Taylor, D. Tody. SAMP-Simple Application Messaging Protocol, [Online], Available: http://www.ivoa.net/Documents/SAMP/20120411/ RECSAMP-1.3-20120411.html, 2013.

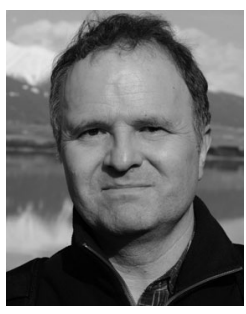

Petr Šaloun graduated and passed the rigorous exam at the Faculty of Science Palacky University in Olomouc, Czechoslovakia in 1986. He received his $\mathrm{Ph}$. D. degree in computer science and engineering at the Czech Technical University, Prague in 2002. $\mathrm{He}$ is an associate professor in computer science at the FEI VSB-Technical University Ostrava, Czech Republic. During his career, he published tens of articles in the proceedings of both domestic and foreign professional and research conferences. He is the author of three books and four university textbooks. He also collaborated on a number of research and educational projects in the field. He is an ACM professional member and an IEEE CS member. He is also a member of research group Unconventional Algorithms and Computing.

His research interests include the processing of large data petabyte (astromical observation), information systems, personalized, adaptive systems, electronic and web publishing technology for e-learning, sequential and parallel parsing of formal languages and compilers and object oriented programming.

E-mail: petr.saloun@vsb.cz (Corresponding author)

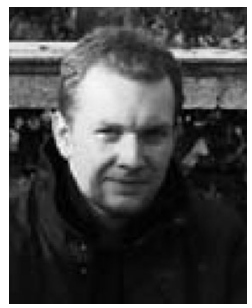

David Andrešič received the B. Sc. degree in computer science at FEI VSBTechnical University Ostrava, Czech Republic in 2013 and will continue his study.

Since 2008, he is programmer and software developer. He is the co-author of an article published in the proceedings of Nostradamus 2013: Prediction, Modeling and Analysis of Complex Systems.

His research interests include production-critical information systems with multi-user access and complex data structures.
E-mail: david.andresic.st@vsb.cz

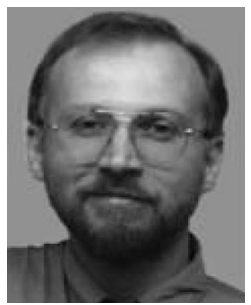

Petr Škoda graduated with honours in astrophysics at the Faculty of Mathematics and Physics, Charles University, Prague, Czechoslovakia in 1987, and received his $\mathrm{Ph}$. D. equivalent title CSc. (Candidatus Scientarum) in astrophysics at the same faculty in 1996. From 1987 to 1989, he was a postgraduate student of the Stellar Department of the Astronomical Institute of the Czechoslovak Academy of Sciences in Ondřejov. Since 1989, he is a staff member there.

He is the author and co-author of more than 100 publications and co-author of several standards of virtual observatory. $\mathrm{He}$ is a member of the International Astronomical Union and several working groups of International Virtual Observatory Alliance (IVOA) as well as an external supervisor and consultant at main Czech faculties of informatics.

His research interests includes the stellar optical spectroscopy, modern astronomical instrumentation in general, big-data realm of astronomical data archives, the virtual observatory and astroinformatics.

E-mail:skoda@sunstel.asu.cas.cz

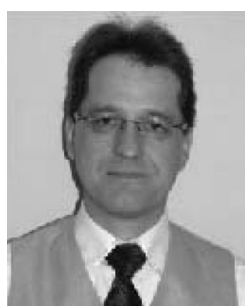

Ivan Zelinka went to the Technical University of Brno, where he studied technical cybernetics and obtained his degree in 1995. He obtained his Ph. D. degree in technical cybernetics at Tomas Bata University, Zlin in 2001. He is an associate professor at the Technical University of Brno and professor position at the VSB-TU Ostrava. He is also a member of British computer society, the chairman of the IEEE Computational Intelligence Czech chapter, head of research group Unconventional Algorithms and Computing.

His research interest include artificial intelligence, bioinspired computation and computer security.

E-mail: ivan.zelinka@vsb.cz 